\title{
Zero-lag long-range synchronization of Hodgkin-Huxley neurons is enhanced by dynamical relaying Raul Vicente ${ }^{* 1,2}$, Gordon Pipa ${ }^{1,2}$, Ingo Fischer ${ }^{3}$ and Claudio R Mirasso ${ }^{4}$
}

\author{
Address: ${ }^{1}$ Department of Neurophysiology, Max-Planck Institute for Brain Research, Frankfurt, Germany, ${ }^{2}$ Frankfurt Institute for Advanced Studies \\ (FIAS), Frankfurt, Germany, ${ }^{3}$ Department of Applied Physics and Photonics, Vrije Universiteit Brussel, Brussel, Belgium and ${ }^{4}$ Departament de \\ Fisica, Universitat de les Illes Balears, Palma de Mallorca, Spain \\ Email: Raul Vicente* - raulvicente@mpih-frankfurt.mpg.de \\ * Corresponding author
}

from Sixteenth Annual Computational Neuroscience Meeting: CNS*2007

Toronto, Canada. 7-12 July 2007

Published: 6 July 2007

BMC Neuroscience 2007, 8(Suppl 2):P42 doi:10.1 I86/I47I-2202-8-S2-P42

(c) 2007 Vicente et al; licensee BioMed Central Ltd.

\section{Background}

The synchrony hypothesis postulates that precise temporal synchronization of different pools of neurons conveys information that is not contained in their firing rates. The synchrony hypothesis had been supported by experimental findings demonstrating that millisecond precise synchrony of neuronal oscillations across well separated brain regions plays an essential role in visual perception and other higher cognitive tasks [1]. Albeit, more evidence is being accumulated in favour of its role as a binding mechanism of distributed neural responses, the physical and anatomical substrate for such a dynamic and precise synchrony, especially zero-lag even in the presence of non-negligible delays, remains unclear [2].

Here we propose a simple network motif that naturally accounts for zero-lag synchronization for a wide range of temporal delays [3]. We demonstrate that zero-lag synchronization between two distant neurons or neural populations can be achieved by relaying the dynamics via a third mediating single neuron or population.

\section{Methods}

We simulated the dynamics of two Hodgkin-Huxley neurons that interact with each other via an intermediate third neuron. The synaptic coupling was mediated through $\alpha$-functions. Individual temporal delays of the arrival of pre-synaptic potentials were modelled by a gamma distribution. The strength of the synchronization and the phase-difference between each individual pairs were derived by cross-correlation of the membrane potentials.

\section{Results}

In the regular spiking regime the two outer neurons consistently synchronize with zero phase lag irrespective of

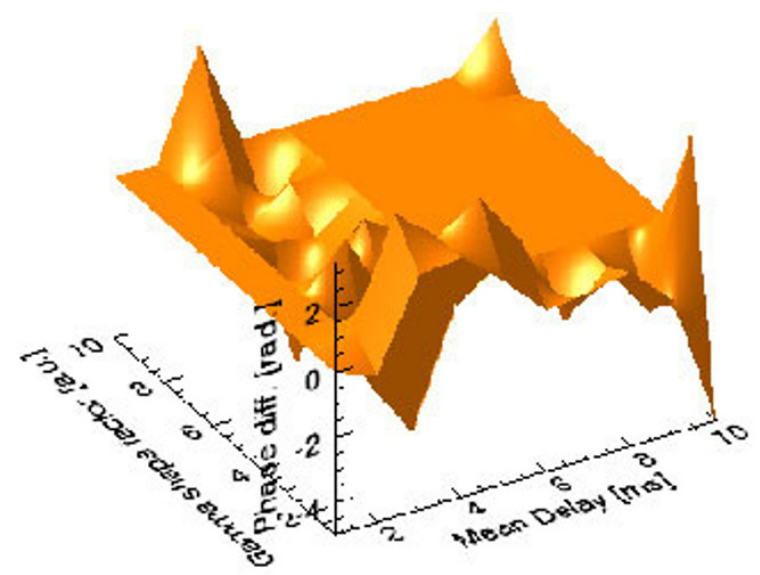

Figure I

Phase difference between the outer neurons as a function of the mean delay and shape factor of the axonal delays distribution. 
the initial conditions. This robust zero-lag synchronization naturally arises as a consequence of the relay and redistribution of the dynamics performed by the central neuron. This result is independent on whether the coupling is excitatory or inhibitory and can be maintained for arbitrarily long time delays (see Fig. 1).

\section{Conclusion}

We have presented a simple and extremely robust network motif able to account for the isochronous synchronization of distant neural elements in a natural way. As opposed to other possible mechanisms of neural synchronization, neither inhibitory coupling, gap junctions nor precise tuning of morphological parameters are required to obtain zero-lag synchronized neuronal oscillation.

\section{References}

I. Varela FJ, Lachaux J-P, Rodriguez E, Martinerie J: The brainweb: phase synchronization and large-scale integration. Nature Reviews Neuroscience 2001, 2:229-239.

2. Singer W: Neuronal synchrony: A versatile code for the definition of relations? Neuron 1999, 24:49-65.

3. Fischer I, Vicente R, Buldu JM, Peil M, Mirasso CR, Torrent MC, Garcia-Ojalvo J: Zero-lag long-range synchronization via dynamical relaying. Phys Rev Lett 2006, 97:123902-.

Publish with Biomed Central and every scientist can read your work free of charge

"BioMed Central will be the most significant development for disseminating the results of biomedical research in our lifetime. "

Sir Paul Nurse, Cancer Research UK

Your research papers will be:

- available free of charge to the entire biomedical community

- peer reviewed and published immediately upon acceptance

- cited in PubMed and archived on PubMed Central

- yours - you keep the copyright

Submit your manuscript here:

http://www.biomedcentral.com/info/publishing_adv.asp 